\title{
In der Form ein unmögliches Amt!
}

VB verfassungsblog.de/der-form-ein-unmgliches-amt/

Hans Michael Heinig Sa 18 Feb 2012

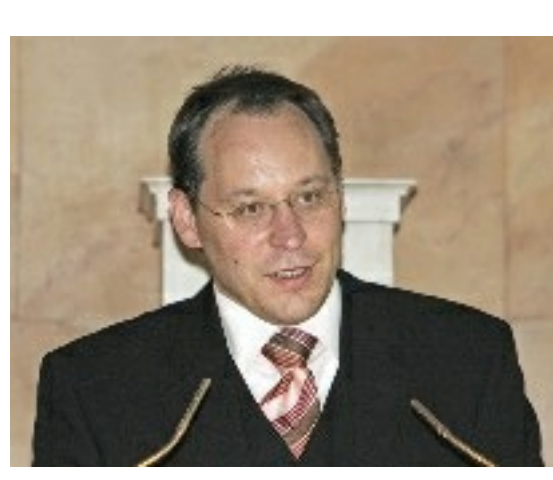

Von HANS MICHAEL HEINIG

Christian Wulff ist zurückgetreten. „Endlich!“, werden wohl die meisten Bürger denken. Bundeskanzlerin Merkel hat angekündigt, koalitionsübergreifende Gespräche über die Neubesetzung anzustreben. Sie kommt damit einer weit verbreiteten Sehnsucht entgegen - der Sehnsucht nach einem Bundespräsidenten als Macht über den Parteien, als Garant des „echten“ Gemeinwohls jenseits aller Partikularinteressen. Doch ist nun wirklich die Suche nach dem „Superpolitiker Herkules“ angesagt, einer Person, hinter der sich die politische Klasse und das gemeine Volk, RTL 2 wie F.A.Z. versammeln können?

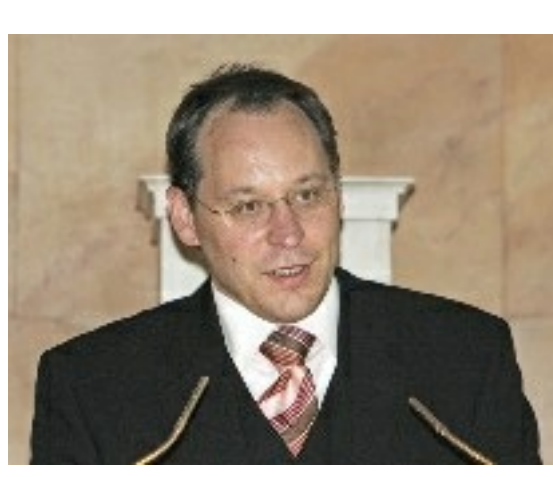

Von HANS MICHAEL HEINIG

Christian Wulff ist zurückgetreten. „Endlich!“, werden wohl die meisten Bürger denken. Bundeskanzlerin Merkel hat angekündigt, koalitionsübergreifende Gespräche über die Neubesetzung anzustreben. Sie kommt damit einer weit verbreiteten Sehnsucht entgegen - der Sehnsucht nach einem Bundespräsidenten als Macht über den Parteien, als Garant des „echten“ Gemeinwohls jenseits aller Partikularinteressen. Doch ist nun wirklich die Suche nach dem „Superpolitiker Herkules“ angesagt, einer Person, hinter der sich die politische Klasse und das gemeine Volk, RTL 2 wie F.A.Z. versammeln können?

Das Grundgesetz gewährt für die Neuwahl 30 Tage Zeit (Art. 54 IV S. 1 2. HS GG). Zwei, drei dieser Tage sollten wir nach der Erfahrung zweier evidenter Fehlbesetzungen vielleicht doch dafür nutzen, noch einmal darüber nachzudenken, wofür so ein Bundespräsident überhaupt noch gut ist.

Dieter Grimm hatte jüngst darauf aufmerksam gemacht, dass das Wesentliche am Amt des Bundespräsidenten nicht seine verfassungsrechtlichen Kompetenzen seien. Dem wird niemand ernsthaft widersprechen wollen. Gerade deshalb stellt sich aber die Frage, ob überhaupt noch jemand die Rollen ausfüllen kann, die dem Amtsträger jenseits seiner Kompetenzen nach dem Grundgesetz zugedacht werden. Ist die Gesellschaft nicht viel zu diversifiziert und zu schnelllebig, ist die politische Kommunikation nicht viel zu amorph, die Komplexität eines europäischen MehrEbenen-Systems nicht viel zu hoch, die Spannbreite zwischen medialem Glamourbedarf und Erwartungen an die präsidiale Pastoralmacht nicht viel zu groß, um den (vermeintlichen) Integrationsbedarf einer Gesellschaft über den Kniff eines kompetenzarmen Staatsoberhauptes auch nur teilweise zu befriedigen?

Die Institution des Bundespräsidenten hatte, in idealisierter Übersteigerung, seine große Zeiten: als Korrektiv zur Kanzlerdemokratie Adenauers (Heuss), als Vorbote des Reifezeugnisses eines demokratischen Machtwechsels (Heinemann), als geschichtspolitischer Praeceptor Germaniae (von Weizsäcker). Doch seit von Weizsäckers Abschied ist das Präsidentenamt über weite Strecken doch nur noch ein müder Abglanz dessen, was wir wünschten, das es wäre und glauben, was es einmal war. Nicht von ungefähr gehören Herzogs „Ruck-Rede“ und Köhlers Warnung vor den „Monstern“ zum festen Bestandteil des politischen Kabaretts. Einzig Johannes Raus Auftritt vor der Knesset hellt die Bilanz ein wenig auf. Doch ansonsten? Wer kann sich nur an das Thema der 3., 4., 5. Berliner Rede erinnern? 
Manche meinen, mit einem Bundespräsidenten Gauck wäre es ganz anders gekommen. In der Tat hat das linksliberale juste milieu Christian Wulff nie verziehen, einen Philosophenersatzkönig im Bellevue verhindert zu haben. Doch bei aller Hochachtung vor der rhetorischen Kraft und denkerischen Klarheit Joachim Gaucks - der Obama-Effekt, die Enttäuschung großer Erwartungen, wäre wohl unvermeidlich gewesen. Gauck selbst hat das gespürt und deutlich artikuliert.

Möglichweise ist die kleine Verfallsgeschichte des Präsidentenamtes also nicht (nur) einem Mangel an Chrisma, politischer Professionalität und moralischer Redlichkeit der Amtsinhaber oder der kulturpessimistischen Grundhaltung ihrer Beobachter geschuldet, sondern schlicht der gesellschaftlichen Unmöglichkeit, ein politisches Amt auszufüllen, dessen Wesen und Auftrag im undefinierten Niemandsland jenseits des durch rechtliche Verfahren und Kompetenzen mühsam pazifizierten Kampfes um politische Macht liegen soll.

Demokratie heißt, Integration durch den kanalisierten Konflikt zwischen politischen Alternativen. Sechzig Jahre Erfahrung mit unseren demokratischen Institutionen legen es nahe, sich mit dieser Form von Integration zu begnügen. Verfassungsrechtlich führt das zu einer einfachen Lösung: Die verfassungsrechtlichen Kompetenzen des Bundespräsidenten übernimmt der Bundesratspräsident. Dank des üblichen Rotationsprinzips wird die Repräsentationskraft des Staatsoberhauptes deutlich gestärkt. Und die Welt erklären und wider die Bundesregierung löcken kann auch der Bundestagspräsident vortrefflich, wie der gegenwärtige Amtsinhaber zeigt.

30 Tage - das ist nicht nur der von der Verfassung gesetzte Zeitraum für die Neuwahl des Bundespräsidenten, sondern auch genug Zeit auch für seine Abschaffung!

Der Verfasser lehrt Öffentliches Recht und Kirchenrecht an der Georg-August-Universität Göttingen.

Das Grundgesetz gewährt für die Neuwahl 30 Tage Zeit (Art. 54 IV S. 12 . HS GG). Zwei, drei dieser Tage sollten wir nach der Erfahrung zweier evidenter Fehlbesetzungen vielleicht doch dafür nutzen, noch einmal darüber nachzudenken, wofür so ein Bundespräsident überhaupt noch gut ist.

Dieter Grimm hatte jüngst darauf aufmerksam gemacht, dass das Wesentliche am Amt des Bundespräsidenten nicht seine verfassungsrechtlichen Kompetenzen seien. Dem wird niemand ernsthaft widersprechen wollen. Gerade deshalb stellt sich aber die Frage, ob überhaupt noch jemand die Rollen ausfüllen kann, die dem Amtsträger jenseits seiner Kompetenzen nach dem Grundgesetz zugedacht werden. Ist die Gesellschaft nicht viel zu diversifiziert und zu schnelllebig, ist die politische Kommunikation nicht viel zu amorph, die Komplexität eines europäischen MehrEbenen-Systems nicht viel zu hoch, die Spannbreite zwischen medialem Glamourbedarf und Erwartungen an die präsidiale Pastoralmacht nicht viel zu groß, um den (vermeintlichen) Integrationsbedarf einer Gesellschaft über den Kniff eines kompetenzarmen Staatsoberhauptes auch nur teilweise zu befriedigen?

Die Institution des Bundespräsidenten hatte, in idealisierter Übersteigerung, seine große Zeiten: als Korrektiv zur Kanzlerdemokratie Adenauers (Heuss), als Vorbote des Reifezeugnisses eines demokratischen Machtwechsels (Heinemann), als geschichtspolitischer Praeceptor Germaniae (von Weizsäcker). Doch seit von Weizsäckers Abschied ist das Präsidentenamt über weite Strecken doch nur noch ein müder Abglanz dessen, was wir wünschten, das es wäre und glauben, was es einmal war. Nicht von ungefähr gehören Herzogs „Ruck-Rede“ und Köhlers Warnung vor den „Monstern“ zum festen Bestandteil des politischen Kabaretts. Einzig Johannes Raus Auftritt vor der Knesset hellt die Bilanz ein wenig auf. Doch ansonsten? Wer kann sich nur an das Thema der 3., 4., 5. Berliner Rede erinnern?

Manche meinen, mit einem Bundespräsidenten Gauck wäre es ganz anders gekommen. In der Tat hat das linksliberale juste milieu Christian Wulff nie verziehen, einen Philosophenersatzkönig im Bellevue verhindert zu haben. Doch bei aller Hochachtung vor der rhetorischen Kraft und denkerischen Klarheit Joachim Gaucks - der Obama-Effekt, die Enttäuschung großer Erwartungen, wäre wohl unvermeidlich gewesen. Gauck selbst hat das gespürt und deutlich artikuliert. 
Möglichweise ist die kleine Verfallsgeschichte des Präsidentenamtes also nicht (nur) einem Mangel an Chrisma, politischer Professionalität und moralischer Redlichkeit der Amtsinhaber oder der kulturpessimistischen Grundhaltung ihrer Beobachter geschuldet, sondern schlicht der gesellschaftlichen Unmöglichkeit, ein politisches Amt auszufüllen, dessen Wesen und Auftrag im undefinierten Niemandsland jenseits des durch rechtliche Verfahren und Kompetenzen mühsam pazifizierten Kampfes um politische Macht liegen soll.

Demokratie heißt, Integration durch den kanalisierten Konflikt zwischen politischen Alternativen. Sechzig Jahre Erfahrung mit unseren demokratischen Institutionen legen es nahe, sich mit dieser Form von Integration zu begnügen. Verfassungsrechtlich führt das zu einer einfachen Lösung: Die verfassungsrechtlichen Kompetenzen des Bundespräsidenten übernimmt der Bundesratspräsident. Dank des üblichen Rotationsprinzips wird die Repräsentationskraft des Staatsoberhauptes deutlich gestärkt. Und die Welt erklären und wider die Bundesregierung löcken kann auch der Bundestagspräsident vortrefflich, wie der gegenwärtige Amtsinhaber zeigt.

30 Tage - das ist nicht nur der von der Verfassung gesetzte Zeitraum für die Neuwahl des Bundespräsidenten, sondern auch genug Zeit auch für seine Abschaffung!

Der Verfasser lehrt Öffentliches Recht und Kirchenrecht an der Georg-August-Universität Göttingen.

\section{LICENSED UNDER CC BY NC ND}

SUGGESTED CITATION Heinig, Hans Michael: In der Form ein unmögliches Amt!, VerfBlog, 2012/2/18, http://verfassungsblog.de/der-form-ein-unmgliches-amt/. 